\title{
Heart Failure with Mid-Range Ejection Fraction - State of the Art
}

\author{
Evandro Tinoco Mesquita, ${ }^{([)}$Letícia Mara dos Santos Barbetta, ${ }^{(0)}$ Eduardo Thadeu de Oliveira Correia ${ }^{(0)}$
}

Hospital Universitário Antônio Pedro, Niterói, RJ - Brazil

\begin{abstract}
In 2016, the European Society of Cardiology (ESC) recognized heart failure $(\mathrm{HF})$ with ejection fraction between 40 and $49 \%$ as a new HF phenotype, HF with mid-range ejection fraction (HFmrEF), with the main purpose of encouraging studies on this new category. In 2018, the Brazilian Society of Cardiology adhered to this classification and introduced HFmrEF in Brazil. This paper presents a narrative review of what the literature has described about HFmrEF. The prevalence of patients with HFmrEF ranged from 13 to $24 \%$ of patients with HF. Analyzing the clinical characteristics, HFmrEF shows intermediate characteristics or is either similar to $\mathrm{HF}$ with preserved ejection fraction (HFpEF) or to HF with reduced fraction (HFrEF). Regarding the prognosis, HFmrEF's all-cause mortality is similar to HFpEF's and lower than HFrEF's. Studies that analyzed cardiac mortality concluded that there was no significant difference between HFmrEF and HFrEF, both of which were lower than HFpEF. Despite the significant increase of publications on HFmrEF, there is a great scarcity of prospective studies and clinical trials that allow delineating specific therapies for this new phenotype. To better treat HFmrEF patients, it is fundamental that cardiologists and internists understand the differences and similarities of this new phenotype.
\end{abstract}

\section{Introduction}

The classification and characterization of heart failure (HF) by phenotypes has an important relevance in clinical practice, since these phenotypes are currently based on left ventricular ejection fraction (LVEF) and have different characteristics in relation to prognosis and treatment. ${ }^{1}$

Classically, two main HF phenotypes have been described; the HF with reduced ejection fraction (HFrEF) with LVEF $<40 \%$ and the HF with preserved ejection fraction (HFpEF), with LVEF $\geq 50 \% .^{2-4}$ Different guidelines have proposed a new phenotype in the current decade, the HF with mid-range ejection fraction (HFmrEF).

The American College of Cardiology/American Heart Association published a new HF guideline in 2013, in which

\section{Keywords}

Heart Failure/physiopathology; Stroke Volume; Natriuretics Peptides; Diagnostic Imaging; Electrocardiography; Ecocardiography; Magnetic Resonance Imaging.

Mailing Address: Letícia Mara dos Santos Barbetta •

Hospital Universitário Antônio Pedro - Av. Marquês do Paraná, 303. Postal

Code 24033-900, Niterói, RJ - Brazil

E-mail: leticiabarbetta@gmail.com

Manuscript received August 20, 2018, revised manuscript December 26, 2018, accepted February 13, 2019

DOI: 10.5935/abc.20190079 patients with LVEF between $41 \%$ and $50 \%$ were classified as "borderline" HFpEF.2 In 2016, the ESC recognized HF with LVEF between $40 \%$ and $49 \%$ as a distinct phenotype; the HFmrEF, mainly intended to stimulate studies that address epidemiology, etiology, characteristics, and prognostics of this new category. ${ }^{3}$ Finally, the Brazilian Society of Cardiology (BSC) introduced HFmrEF as a new clinical phenotype in its 2018 guideline of acute and chronic HF. ${ }^{5}$

With the introduction of this new classification, HFmrEF has received great attention and, consequently, has been better studied and characterized. The present review study aims to describe what is currently known about HFmrEF and discuss future perspectives that will contribute to a better approach for this group of patients.

\section{Epidemiology}

\section{Prevalence}

In the United States, it is estimated that more than 6.5 million people have $\mathrm{HF}^{6}$ and the percentage of individuals with HFmrEF is between $13 \%$ and $24 \%{ }^{7,8}$ The prevalence of HFmrEF in studies performed with hospitalized patients ranged from $13 \%$ to $26 \%,{ }^{7,9-12}$ while the prevalence of HFmrEF in outpatients varied from $9 \%$ to $21 \%{ }^{8,13-17}$

The last census of Brazilian Institute for Geography and Statistics (IBGE) in 2010 census showed an increase in the elderly population in Brazil, and therefore a great potential for the increase of at-risk HF patients. In the DIGITALIS study performed in the city of Niterói, state of Rio de Janeiro, Brazil, a prevalence of $9.3 \%$ of HF was identified in patients from the family physician program (59 individuals among 633 volunteers), ${ }^{18}$ in which $64.2 \%$ of these patients were characterized as having HFpEF and $35 \%$ as HFrEF. ${ }^{18}$ Recently, according to unpublished data based on the DIGITALIS study database, the prevalence of HFmrEF patients in Niterói was 22\%, HFrEF was 19\% and HFpEF was 59\%.

\section{Diagnosis}

According to the latest acute $\mathrm{HF}$ guideline of $\mathrm{BSC}_{1}{ }^{5}$ the diagnosis of $\mathrm{HF}$ is based on the combination on medical history findings, physical examination, electrocardiogram and chest $x$-ray results, as detailed in figure 1 . An echocardiogram should be performed for diagnostic confirmation if there is clinical suspicion of HF. In low suspicion cases or if there are diagnostic doubts, the measurement of brain natriuretic peptides (BNP and/or NT-proBNP) and an echocardiogram should be performed, if available. A normal echocardiogram and/or plasma BNP levels $<35 \mathrm{pg} / \mathrm{mL}$ and/or NT-proBNP $<125 \mathrm{pg} / \mathrm{mL}$ make the HF diagnosis improbable. In the presence of BNP levels > $35 \mathrm{pg} / \mathrm{mL}$ and/or NT-proBNP > $125 \mathrm{pg} / \mathrm{mL}$ and/or altered echocardiogram results, the HF diagnosis becomes probable. The LVEF echocardiography evaluation contributes 


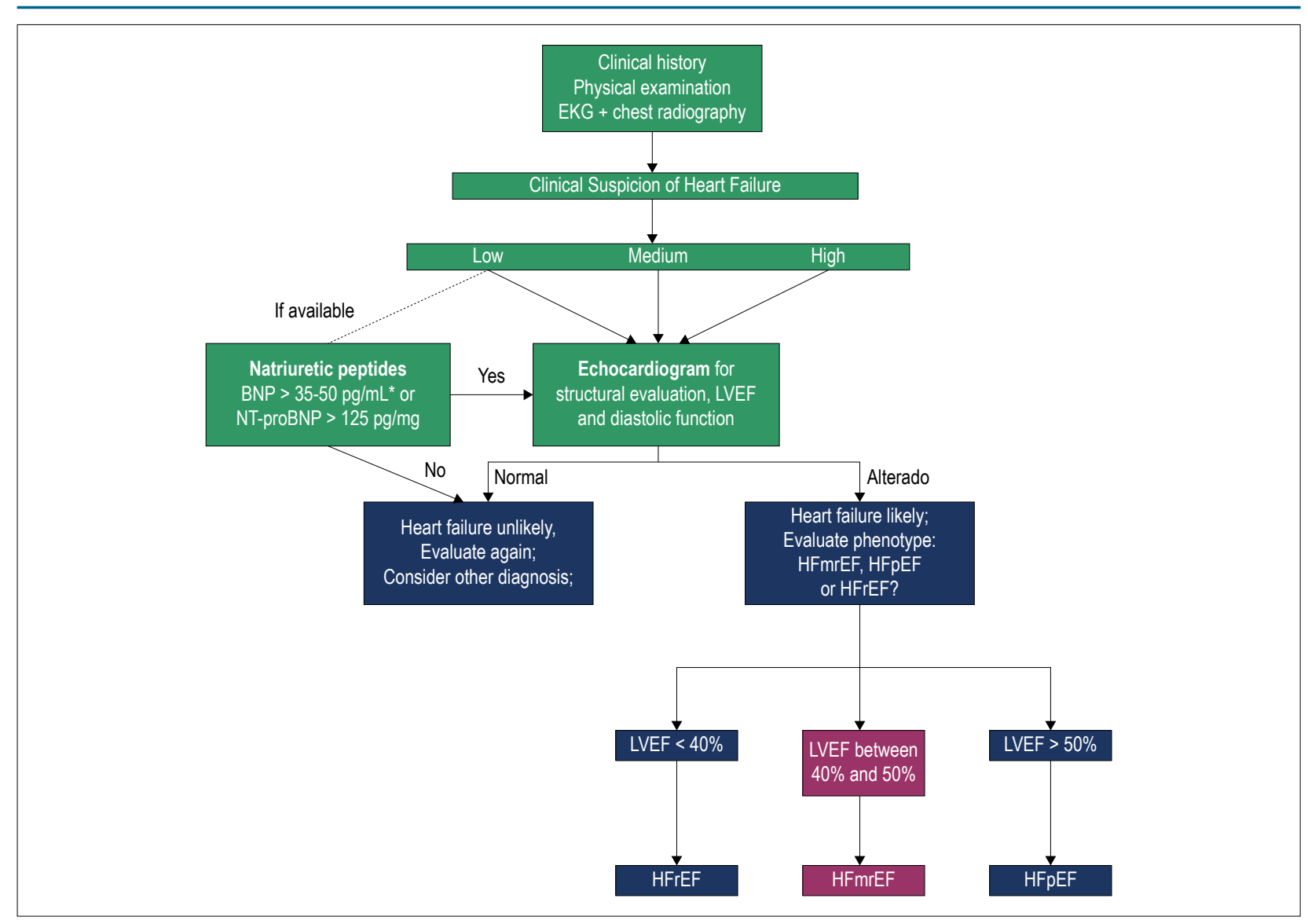

Figure 1 - Diagnostic algorithm in the clinical suspicion of heart failure. Adapted from: Brazilian Guideline for Chronic and Acute Heart Failure of 2018; ${ }^{5} \mathrm{HFrEF}$ : heart failure with reduced ejection fraction; HFmrEF: heart failure with mid-range ejection fraction; HFpEF: heart failure with preserved ejection fraction; EKG: electrocardiogram; BNP: brain natriuretic peptide; NT-proBNP: amino-terminal fragment of pro-brain natriuretic peptide; LVEF: left ventricular ejection fraction.

to establishing the HF clinical phenotype, since the clinical signs and patients' symptoms with HFrEF, HFmrEF and HFpEF are similar. ${ }^{3}$

A relevant aspect regarding the HFmrEF diagnosis involves methodological aspects related to the cardiac imaging techniques. The LVEF evaluation by echocardiography has been the standard method used to categorize patients with HF; however, it is common that the values obtained are different in relation to other methods, such as cardiac magnetic resonance imaging, radioisotope ventriculography and angiocardiography. ${ }^{19,20}$ In addition, the ejection fraction evaluation by echocardiography shows considerable intra and inter-observer variability over time, as well as under therapeutic intervention effect. ${ }^{19,20}$

\section{Clinical-epidemiological characteristics}

Previous studies have shown that patients with HFmrEF had clinical characteristics that, although intermediate between the HFrEF and HFpEF groups, were more similar to those of HFpEF. ${ }^{8,9,13,21}$ Nevertheless, in relation to the presence of ischemic disease, different studies have found that HFmrEF resembles HFrEF, showing a higher prevalence. ${ }^{\text {,22-24 }}$
In the study by Kapoor et al., ${ }^{7}$ based on the GWTG-HF (Get With The Guidelines - Heart Failure) registry, patients with HFmrEF were older (mean age of seventy-seven years) and showed a higher percentage of females (48\%) when compared to patients with HFrEF, being more similar to HFpEF. Moreover, HFmrEF showed a high prevalence of comorbidities such as DM (50\%), atrial fibrillation (AF) $(42 \%)$, chronic obstructive pulmonary disease (COPD) $(36 \%)$, anemia (27\%) and renal failure (26\%), "also similar to HFpEF to HFpEF. However, there was a higher prevalence of ischemic heart disease in up to two thirds of the patients, similar to what as observed with HFrEF.

However, in the meta-analysis published by Lauritsen et al., ${ }^{25}$ patients with HFmrEF had entirely intermediate characteristics, and there were significant differences between patients with HFmrEF and HFrEF and between patients with HFmrEF and HFpEF. Patients with HFmrEF were older than those with HFrEF $(p<0.001)$ but were younger than those with HFpEF $(p<0.001)$. The proportion of men and the prevalence of ischemic heart disease in patients with HFmrEF were lower than in those with $\operatorname{HFpEF}(p<0.001$ and $p<0.034$, respectively), but higher than in those with HFpEF $(p<0.001$ and $p<0.034$ respectively). Hypertension was more frequent in patients with 
HFmrEF than in those with HFrEF $(p<0.001)$, but less frequent than in patients with HFpEF ( $p<0.001)$. Diabetes mellitus (DM) was significantly less frequent in patients with HFmrEF and HFrEF $(p=0.17)$ than in those with HFpEF $(p=0.021)$. AF was more frequent among patients with HFmrEF than among those with HFrEF $(p<0.001)$, but less frequent than in patients with HFpEF $(p<0.001)$. The prevalence of COPD was lower in individuals with HFmrEF than in HFpEF ( $p<0.001)$, but higher when compared to patients with HFrEF ( $p=0.001)$. Patients with HFmrEF had significantly better renal function than patients with HFpEF ( $p<0.001)$ but worse than patients with $\operatorname{HFrEF}(p=0.001)$.

In the RICA $^{26}$ registry, patients with HFmrEF showed mixed characteristics in relation to the other groups. Patients with HFmrEF were similar to patients with HFrEF regarding hypertension rates, and chronic kidney disease (CKD) history, as well as in relation to the presence of higher systolic pressure, higher blood pressure, lower frequency of New York Association (NYHA) classes III-IV, higher prevalence of $\mathrm{AF}$ and previous $\mathrm{HF}$.

The study by Bhambhani et al., ${ }^{22}$ which analyzed 28,829 without HF participants for an average of 12 years, found that $48 \%$ of the patients who developed HFmrEF were females. In addition, participants with HFmrEF shared some similarities with the HFrEF group, including lower body mass index (BMI) in relation to patients with HFpEF, with a lower obesity prevalence, a higher coronary artery disease (CAD) prevalence and lower levels of high density lipoproteins (HDL). Other clinical characteristics of participants with HFmrEF were intermediate between those with HFpEF and HFrEF.

The $\mathrm{CHARM}^{27}$ study found that patients with HFmrEF were similar to HFpEF for most of the characteristics, including age, systolic blood pressure, percentage of women, previous myocardial infarction and AF. HFmrEF was intermediate between HFrEF and HFpEF regarding the history of hypertension, NYHA and BMI class distribution.
Some characteristics, such as DM, were simultaneously prevalent in all three categories..$^{27,28}$

In addition, the study by Wang et al..$^{23}$ showed no significant differences in gender between HFmrEF, HFpEF and HFrEF. The HFmrEF group was intermediate compared to the other groups regarding characteristics such as age, smoking history, DM and CKD. In contrast, the HFmrEF group was similar to $\mathrm{HFpEF}$ regarding the history of ischemic heart disease, with both groups showing significantly higher rates than HFpEF.

In the Swedish Heart Failure ${ }^{24}$ registry, HFmrEF was intermediate in terms of age, gender, hypertension, AF, valvular and renal disease. However, the presence of ischemic disease was more common in HFrEF and HFmrEF when compared to HFpEF, and the prevalence of DM did not differ between the three groups. The BMI was lower and fewer patients had anemia in HFmrEF.

A summary of the clinical-epidemiological HFmrEF characteristics is shown in figure 2 .

\section{Biomarkers}

Regarding biomarkers, HFmrEF has an intermediate profile, with inflammatory biomarkers being more common in HFpEF and heart distension biomarkers in HFrEF. ${ }^{13}$ In the study by Bhambhani et al. ${ }^{22}$ was found that the predictors of HFmrEF were similar to the predictors of other types of HF. However, a higher BMI was a predictor of HFpEF, but not of HFmrEF, and natriuretic peptides were more robust predictors of HFpEF than of HFmrEF.

The Swedish Heart Failure registry ${ }^{24}$ concluded the median value of NT-pro BNP in HFmrEF was 1,540 pg / $\mathrm{mL}$ with an interquartile range of $652-3,317$. This value was minimally and not significantly higher than in HFpEF but was significantly higher than in HFrEF $(p<0.001)$. The study by Moliner et al..$^{29}$ also concluded that NT-ProBNP levels in HFmrEF were significantly lower than in $\operatorname{HFrEF}(p=0.02)$, but similar to

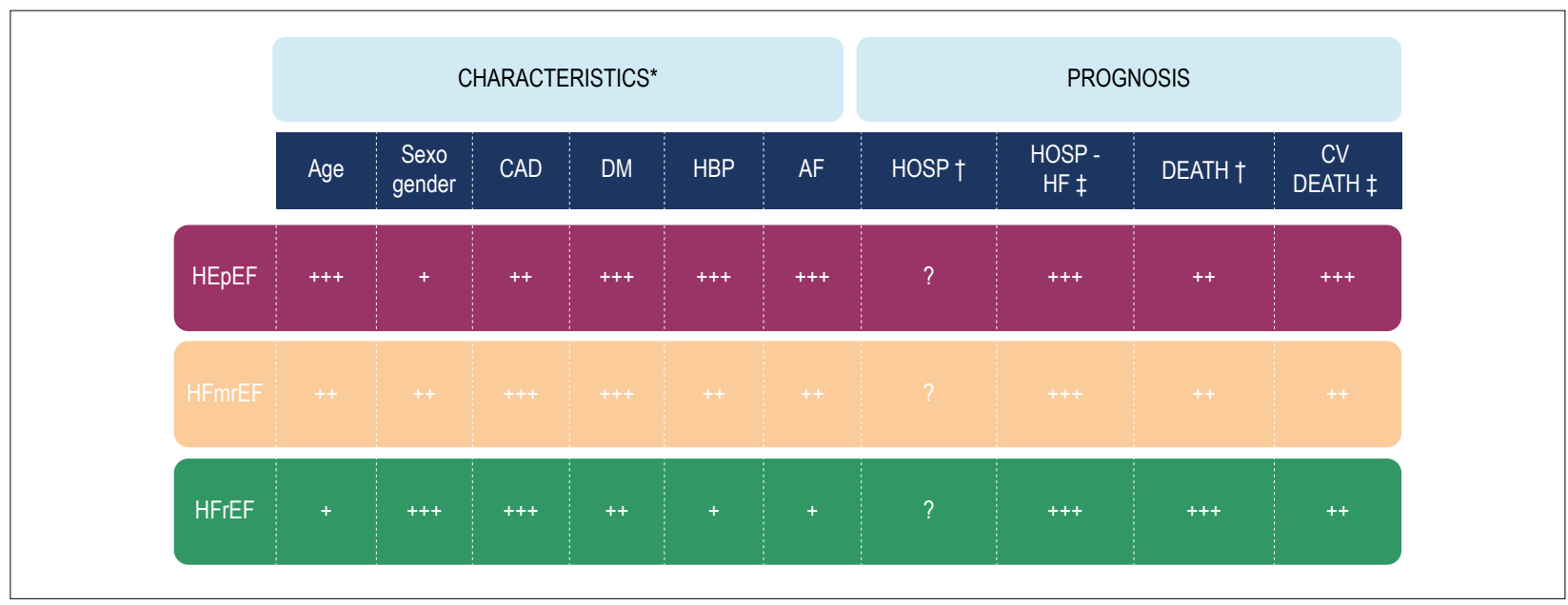

Figure 2 - Comparisons of the clinical characteristics among the different phenotypes of HF; ? : presence of conflict between studies; CAD: coronary artery disease; DM: diabetes mellitus; HBP: high blood pressure (hypertension); AF: atrial fibrillation; HOSP: hospitalization; HOSP-HF: hospitalization for HF; DEATH: death from all causes; CV-DEATH: cardiovascular death; * Data for constructing the characteristics were taken from references, ${ }^{7,22-27,32}+$ Data taken from references, ${ }^{27,32} \ddagger D$ ata taken from reference. ${ }^{32}$ 


\section{Review Article}

HFpEF levels $(p=0.88)$. All other biomarkers were similar between HFrEF and HFmrEF. Cystatin-C and ST2 were significantly lower in HFmrEF than in HFpEF $(p=0.01$ and $p=0.02$, respectively) Galectin-3 and soluble transferrin receptor were relatively lower in HFmrEF when compared to HFpEF, but the difference was not statistically significant.

\section{Pathophysiology}

In the 2016 guideline, the ESC suggested that HFmrEF may have both a mild systolic dysfunction and a diastolic dysfunction contribution. ${ }^{3}$ A recent study published by Rastogi et al., ${ }^{30}$ observed that HFmrEF consists of a heterogeneous group of patients, and consists of at least 3 subgroups based on LVEF, such as: patients with previous LVEF $<40 \%$ (recovered HFmrEF), patients with previous LVEF $>50 \%$ (impaired HFmrEF) and patients with previous LVEF between $40-50 \%$ (unchanged HFmrEF). ${ }^{30}$ Most patients in this study were classified as having recovered $\mathrm{HFmrEF} \mathrm{(73 \% ),} \mathrm{while}$ $17 \%$ of patients were classified as impaired HFmrEF and only $10 \%$ were categorized as unchanged $\mathrm{HFmrEF}^{30}$

Also, in this study, the subgroup with recovered HFmrEF had a higher prevalence of male patients and a higher prevalence of patients with CAD, compatible with the characteristics of patients with HFrEF. In contrast, the subgroup with impaired HFmrEF consisted mostly of women with a history of hypertension and AF or flutter, as well as patients with HFpEF. In contrast, the subgroup with impaired HFmrEF consisted mostly of women with a history of hypertension and AF or flutter, as well as patients with HFpEF. Another important observation, in the impaired HFmrEF subgroup, patients had significantly more advanced diastolic dysfunction at the echocardiogram assessment when compared to patients with recovered $\mathrm{HFmrEF}^{30} \mathrm{~A}$ common finding in different cohorts ${ }^{13,14,31}$ was that HFmrEF resembled HFrEF in relation to the high prevalence of CAD and a higher risk of new CAD events. In the Swedish Heart Failure register, no difference was observed between the prevalence of CAD between HFmrEF (61\%) and HFrEF (60\%), while HFpEF was associated with a lower prevalence of the disease (52\%). ${ }^{14}$ Chioncel et al. ${ }^{31}$ based on the long-term HF record of the ESC, found that ischemic etiology was present in $48.6 \%$ of patients with HFrEF, $41.8 \%$ of patients with HFmrEF, but only in $23.7 \% \%$ of patients with HFrEF. In the TIME-CHF study, ${ }^{24}$ post-hoc analysis, the ischemic etiology was $58.2 \%$, $56.5 \%$ and $31.3 \%$ for HFrEF, HFmrEF and HFpEF, respectively. Therefore, regarding the etiology, patients with HFmrEF are more similar to those with HFrEF than to the ones with HFpEF.

\section{Prognosis}

Both the CHARM study and the prognosis meta-analysis performed by Altaie et al. ${ }^{32}$ concluded that all-cause mortality in HFmrEF patients is significantly lower than in patients with HFrEF $(p<0.001$ and RR 0.9; 95\% Cl 0.85-0.94; $p<0.001$, respectively) and statistically similar to patients with (HR 0.98; Cl 95\% $0.82-1.19 ; \mathrm{p}=0.88$ and RR 0.98; 95\% Cl 0.86-1.12; $\mathrm{p}=0.82$, respectively). ${ }^{27,32}$

Regarding the cardiac mortality, the meta-analysis of Altaie et al. ${ }^{32}$ concluded there was no significant difference between
HFrEF and HFmrEF (RR 0.89, 95\% Cl, 0.69-1.15, p = 0.38) while HFpEF showed significantly higher cardiac mortality (RR 1.09, 95\% Cl, 1.02-1.16, $\mathrm{p}=0.001$ ).

In the analysis of the prognosis by separating subgroups of HFmrEF, in the study by Rastogi et al., ${ }^{30}$ the patient cohort with recovered HFmrEF showed significantly better clinical outcomes compared to patients with HFrEF, after adjusting for age and gender. In contrast, the clinical endpoints of the subgroup with impaired HFmrEF were not significantly different from those with HFpEF after adjusted for the same factors. ${ }^{30}$ By observing time to death / transplantation / cardiac hospitalization between the subgroups, the recovered HFmrEF had a significantly better prognosis compared to impaired HFmrEF ( $p=0.011)$, whereas there was no significant difference between the two groups and unchanged HFmrEF. ${ }^{30}$

\section{Hospitalization}

The studies differed regarding hospitalization rates. The meta-analysis of Altaie et al., ${ }^{32}$ demonstrated that there was no significant difference in all-cause hospitalization for both HFrEF and HFmrEF, and between HFpEF and HFmrEF (RR 0.91, 95\% $\mathrm{Cl}, 0.18-4.59, \mathrm{p}=0.9$, and $\mathrm{RR} 0.95,95 \% \mathrm{Cl}, 0.84-1.07$; $\mathrm{p}=0.38$, respectively). Regarding the HF hospitalization, the meta-analysis also did not show any significant differences between HFrEF and HFmrEF or between HFpEF and HFmrEF (RR 0.92, 95\% Cl, 0.84-1.01, p = 0.08, and RR 1.05, 95\% $\mathrm{Cl}, 0.83-1.33 ; \mathrm{p}=0.69$, respectively.) However, in the CHARM study, all-cause hospitalization was significantly lower in patients with HFmrEF than in the HFpEF phenotype ( $\mathrm{HR} 8.89 ; 95 \% \mathrm{Cl}, 0.81-0.98 ; \mathrm{p}=0.02) .{ }^{27}$ When comparing the different HFmrEF in the Rastogi et al., ${ }^{30}$ cohort subgroups, the recovered HFmrEF had a better prognosis compared to HFmrEF ( $p=0.029$ ) when observing the time until the first hospitalization for a cardiac event. However, there was no significant difference in relation to the subgroup of unchanged HFmrEF when compared to the other two.

\section{Pharmacological treatment and comorbidity management}

In the TOPCAT study, spironolactone did not present in the primary endpoint (consisting of cardiovascular death, cardiac arrest or HF hospitalization), however, there was a reduction in HF hospitalizations in the treatment group with the greatest benefit observed in patients with LVEF from $45 \%$ to $55 \% .^{33}$

On the other hand, the study by Yan-guo Xin et al., ${ }^{34}$ which evaluated spironolactone use in 229 patients with HFmrEF, showed that the drug use reduced the incidence of primiparous death from all causes (21.3\% vs. $34.5 \%, p=0.014)$, as well as improving quality of life. However, there was no difference between the groups receiving different doses of medication (21.8 vs $20.7 \%, p=0.861 .50 \mathrm{mg}$ vs. $25 \mathrm{mg}$, respectively).

The OPTIMIZE-HF study, when evaluating the use of ACE inhibitors and ARBs, showed there was no associated benefit in patients with HFmrEF. ${ }^{21}$ Patients with LVEF $<40 \%$ were compared with those with LVEF $\geq 40 \%$, for long-term outcomes in relation to the use of beta-blockers. ${ }^{21}$ In patients with LVEF of $40-50 \%$, as in all patients with LVEF $\geq 40 \%$, there was no significant influence of drug use on the outcomes. ${ }^{35}$ 
However, the CHARM study showed that the candesartan use improved outcomes for HFmrEF to a degree comparable to improvement for HFrEF. For the HFmrEF group, the incidence rates for the primary outcome (cardiovascular death or HF hospitalization) of candesartan vs. placebo were 7.4 vs. 9.7 per 100 patients per year ( $\mathrm{HR} 0.76,95 \% \mathrm{Cl}, 0.61-0.96, \mathrm{p}=0.02)$, and the incidence rate of recurrent hospitalization for $\mathrm{HF}$ was $0.48(95 \% \mathrm{Cl}, 0.33-0.70, \mathrm{p}<0.001) .{ }^{27,36}$

The study by Cleland JGF et al., ${ }^{37}$ which included 18,637 patients, found that for patients with HF with sinus rhythm and LVEF between $40 \%$ and $49 \%$, beta-blockers showed a reduction in cardiovascular death when compared to placebo ( $\mathrm{HR} 0.048,95 \% \mathrm{Cl}, 0.24-0,97, \mathrm{p}=0.04)$ and improvement in LV systolic function. ${ }^{37}$

In the study by Gwag et al., ${ }^{38}$ maintenance therapy with $\beta$-blocker was seen to be associated with LVEF improvement in patients with HFmrEF (HR 2.021; 95\% HF 1.033-3.033; $\mathrm{p}=0.04)$. In addition, maintenance therapy with reninangiotensin system blockers or aldosterone antagonists were significantly associated with improved survival (HR 0,309; Cl 95\% 0,162-0,588; $\mathrm{p}<0,001$; and HR 0,240; Cl 95\% $0,085-0,673 ; p=0,01$, respectively).

Digoxin use was evaluated in the study by Abdul-Rahim AH et al., ${ }^{39}$ which included 7788 patients, with 1995 patients being classified as HFmrEF. Digoxin reduced cardiovascular death or HF hospitalization (HR: 0.83; 95\% Cl, 0.66-1.05). ${ }^{39}$

The study Chang et al. ${ }^{9}$ showed the comorbidities observed in patients with HFmrEF were more similar to the ones observed in patients with HFpEF, and CAD was associated with greater declines in LVEF in patients with HFpEF. ${ }^{40}$ Therefore, the management of CAD can help prevent LV systolic dysfunction progression in individuals with HFmrEF. ${ }^{21}$
Non-cardiac comorbidities, such as hypertension, DM and COPD, are highly prevalent in the HF population and contribute to the general morbidity of these patients. ${ }^{41}$ In patients with HFmrEF, uncontrolled hypertension was the main precipitant factor of hospitalization for HF compared to the other HF groups. ${ }^{7}$ In patients with HFmrEF and hypertension, therapy with angiotensin II receptor blockers (ARB) or aldosterone antagonists has shown a reduction in hospitalizations, which suggests that such drugs can be used to control hypertension and reduce the risk of LVEF decline in patients with $\mathrm{HFmrEF}^{7}$ Regarding the patients with HF undergoing treatment for DM sodium-glucose cotransporter-2 (SGLT2) inhibitors use in patients at high cardiovascular risk showed improvements in the primary outcome, consisting of death from cardiovascular causes, infarction and non-fatal stroke. (HR 0.86; 95\% Cl, 0.74-0.99; p < 0.001 for noninferiority and $p=0.04$ for superiority). In addition, empagliflozin use showed a reduction in cardiovascular death and death from all causes (HR 0.62, 95\% Cl 0.49-0.77, $\mathrm{p}<0.001$ and HR 0.68, 95\% Cl, 0.57-0.82, p < 0.001, respectively), in addition to the reduction in hospitalization for HF (HR 0.65, 95\% Cl, 0.50-0.85, p = 0.002). ${ }^{42}$

The current BSC HF guideline ${ }^{5}$ proposes that initially, the specific treatment of the etiology and comorbidities should be addressed, when possible. Patients with a history of HFrEF who show an improvement of LVEF, which reclassifies them as HFmrEF patients, should be treated by maintaining the therapeutic optimization for HFrEF. For patients with previous HFpEF who show worsening of LVEF and also those with persistent HFmrEF, the use of beta-blocker and angiotensinconvertingenzyme inhibitor (ACEi) or ARB (if ACEi is not tolerated) is recommended. The treatment scheme proposed by the SBC is shown in figure 3 .

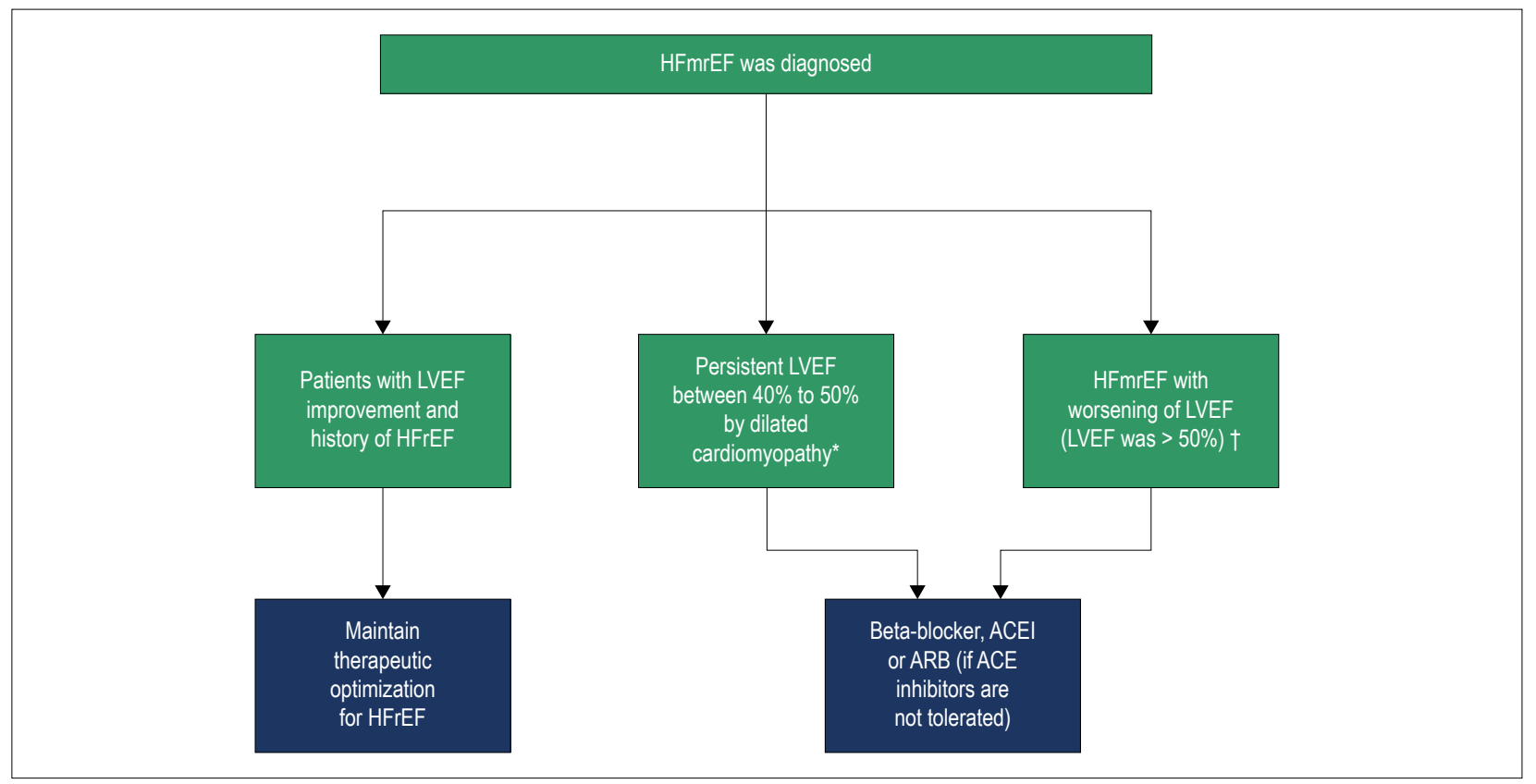

Figure 3 - Algorithm for treatment of HFmrEF according to the Brazilian Guideline for Chronic and Acute Heart Failure of 2018; ${ }^{5}$ ACEi: angiotensin-converting-enzyme inhibitor; ARB: angiotensin II receptor blockers; *In the absence of deposit cardiomyopathies, hypertrophic, inflammatory or infectious diseases; $†$ Particularly for coronary heart disease and/or acute myocardial infarction. 


\section{Review Article}

\section{Future perspectives}

The precision medicine use in the cardiovascular area has advanced, and the identification of HF phenotypes is important for the development of new therapeutic alternatives that offer a better prognosis for the patient with HF.

Although some studies have demonstrated the efficacy of certain therapies in patients with HFmrEF, most publications are retrospective studies that perform a new analysis of previous databases. Therefore, prospective studies and randomized clinical trials including patients with HFmrEF are essential for the creation of therapies with solid evidence-based recommendations.

\section{Conclusion}

After the establishment of HFmrEF as a new HF category by national and international guidelines, there was a considerable increase in publications on this type of patients, which allowed a better understanding of their clinical profile, pathophysiological and clinical outcome. However, there is still a great shortage of prospective studies and randomized double-blind clinical trials that allow the specific therapy delineation for this new category of HF. The knowledge of HFmrEF peculiarities by cardiologists and internists is fundamental for the best diagnosis and management of these patients, in addition to the identification of areas of uncertainty regarding the development of basic and clinical researches.

\section{Author contributions}

Conception and design of the research, Analysis and interpretation of the data, Writing of the manuscript and Critical revision of the manuscript for intellectual content: Mesquita ET, Barbetta LMS, Correia ETO; Acquisition of data: Barbetta LMS, Correia ETO.

\section{Potential Conflict of Interest}

No potential conflict of interest relevant to this article was reported.

\section{Sources of Funding}

There were no external funding sources for this study.

\section{Study Association}

This study is not associated with any thesis or dissertation work.

\section{Ethics approval and consent to participate}

This article does not contain any studies with human participants or animals performed by any of the authors.

\section{References}

1. Wang TJ, Evans JC, Benjamin EJ, Levy D, LeRoy EC, Vasan RS. Natural History of Asymptomatic Left Ventricular Systolic Dysfunction in the Community. Circulation. 2003;108(8):977-82.

2. Yancy CW, Jessup M, Bozkurt B, Butler J, Casey DE Jr, Drazner MH, et al. 2013 ACCF/AHA guideline for the management of heart failure: a report of the American College of Cardiology Foundation/American Heart Association Task Force on Practice Guidelines. J Am Coll Cardiol 2013;62(16):e147-239.

3. Ponikowski P, Voors AA, Anker SD, Bueno H, Cleland JG, Coats AJ, et al. 2016 ESC guidelines for the diagnosis and treatment of acute and chronic heart failure: the Task Force for the diagnosis and treatment of acute and chronic heart failure of the European Society of Cardiology (ESC) developed with the special contribution of the Heart Failure Association (HFA) of the ESC. Eur Heart J 2016;37(27):2129-200.

4. Bocchi EA, Marcondes-Braga FG, Bacal F, Ferraz AS, Albuquerque D Rodrigues D, et al; Sociedade Brasileira de Cardiologia. Atualização da Diretriz brasileira de insuficiência cardíaca crônica - 2012. Arq Bras Cardiol. 2012;98(1 Suppl 1):1-33.

5. Comitê Coordenador da Diretriz de Insuficiência Cardíaca. Diretriz Brasileira de Insuficiência Cardíaca Crônica e Aguda. Arq Bras Cardiol. 2018; 111(3):436-539.

6. Benjamin EJ, Blaha MJ, Chiuve SE, Cushman M, Das SR, Deo R, et al. Heart disease and stroke statistics - 2017 update: a report from the American Heart Association. Circulation. 2017;135(10):e146-603.

7. Kapoor JR, Kapoor R, Ju C, Heidenreich PA, Eapen ZJ, Hernandez AF, et al. Precipitating clinical factors, heart failure characterization, and outcomes in patients hospitalized with heart failure with reduced, borderline, and preserved ejection fraction. JACC Heart Fail. 2016;4(6):464-72.
8. Tsuji K, Sakata Y, Nochioka K, Miura M, Yamauchi T, Onose T, etal. Characterization of heart failure patients with midrange left ventricular ejection fraction-a report from the CHART-2 study. Eur J Heart Fail. 2017; 19(10):1258-69.

9. Cheng RK, Cox M, Neely ML, Heidenreich PA, Bhatt DL, Eapen ZJ, et al. Outcomes in patients with heart failure with preserved, borderline, and reduced ejection fraction in the Medicare population. Am Heart J. 2014; 168(5):721-30.

10. Coles AH, Tisminetzky M, Yarzebski J, Lessard D, Gore JM, Darling CE, et al. Magnitude of and prognostic factors associated with 1-year mortality after hospital discharge for acute decompensated heart failure based on ejection fraction findings. J Am Heart Assoc. 2015;4(10):e002303.

11. Gómez-Otero I, Ferrero-Gregori A, Varela Román A, Seijas Amigo J, PascualFigal DA, et al. Mid-range ejection fraction does not permit risk stratification among patients hospitalized for heart failure. Rev Esp Cardiol (Engl Ed). 2017;70(5):338-46.

12. Farmakis D, Simitsis P, Bistola V, Triposkiadis F, Ikonomidis I, Katsanos S, et al. Acute heart failure with mid-range left ventricular ejection fraction: clinical profile, in-hospital management, and short-term outcome. Clin Res Cardiol. 2017;106(5):359-68.

13. Rickenbacher P, Kaufmann BA, Maeder MT, Bernheim A, Goetschalckx $\mathrm{K}$, Pfister $\mathrm{O}$, et al. Heart failure with mid-range ejection fraction: a distinct clinical entity? Insights from the Trial of Intensified versus standard Medical therapy in Elderly patients with Congestive Heart Failure (TIME-CHF). Eur J Heart Fail. 2017;19(12):1586-96.

14. Vedin O, Lam CSP, Koh AS, Benson L, Teng THK, Tay WT, et al. Significance of ischemic heart disease in patients with heart failure and preserved, midrange, and reduced ejection fraction: a nationwide cohort study. Circ Heart Fail. 2017 Jun;10(6):e003875. 
15. Pascual-Figal DA, Ferrero-Gregori A, Gomez-Otero I, Vazquez R, DelgadoJimenez J, Alvarez-Garcia J, et al. Mid-range left ventricular ejection fraction: clinical profile and cause of death in ambulatory patients with chronic heart failure. Int J Cardiol. 2017 Aug;240:265-70.

16. Ghio S, Guazzi M, Scardovi AB, Klersy C, Clemenza F, Carluccio E, et al. Different correlates but similar prognostic implications for right ventricular dysfunction in heart failure patients with reduced or preserved ejection fraction. Eur J Heart Fail. 2017;19(7):873-9.

17. Allen LA, Magid DJ, Gurwitz JH, Smith DH, Goldberg RJ, Saczynski J, et al. Risk factors for adverse outcomes by left ventricular ejection fraction in a contemporary heart failure population. Circ Heart Fail. 2013;6(4):635-46.

18. Jorge AJ, Rosa ML, Correia DM, Martins WA, Ceron DM, Coelho LC, et al. Evaluation of Quality of Life in Patients with and without Heart Failure in Primary Care. Arq Bras Cardiol. 2017;109(3):248-52.

19. Dunlay SM, Roger VL, Weston SA, Jiang R, Redfield MM. Longitudinal changes in ejection fraction in heart failure patients with preserved and reduced ejection fraction. Circ Heart Fail. 2012;5(6):720-6.

20. Clarke CL, Grunwald GK, Allen LA, Barón AE, Peterson PN, Brand DW, et al. Natural history of left ventricular ejection fraction in patients with heart failure. Circ Cardiovasc Qual Outcomes. 2013;6(6):680-6.

21. Fonarow GC, Stough WG, Abraham WT, Albert NM, Gheorghiade M, Greenberg BH, et al. Characteristics, treatments, and outcomes of patients with preserved systolic function hospitalized for heart failure: a report from the OPTIMIZE-HF Registry. J Am Coll Cardiol. 2007;50(8):768-77.

22. Bhambhani V, Kizer JR, Lima JA, van der Harst P, Bahrami H, Nayor M, et al. Predictors and outcomes of heart failure with mid-range ejection fraction. Eur J Heart Fail. 2018;20(4):651-9.

23. Wang N, Hales S, Barin E, Tofler G. Characteristics and outcome for heart failure patients with mid-range ejection fraction. J Cardiovasc Med (Hagerstown);19(6):297-303.

24. Savarese G, Orsini N, Hage C, Dahlström U, Vedin O, Rosano GM, et al. Associations with and prognostic and discriminatory role of n-terminal pro-b-type natriuretic peptide in heart failure with preserved versus midrange versus reduced ejection fraction. J Card Fail. 2018;24(6):365-74

25. Lauritsen J, Gustafsson F, Abdulla J. Characteristics and long-term prognosis of patients with heart failure and mid-range ejection fraction compared with reduced and preserved ejection fraction: a systematic review and metaanalysis. ESC Heart Fail. 2018;5(4):685-94.

26. Guisado-Espartero ME, Salamanca-Bautista P, Aramburu-Bodas Ó, CondeMartel A, Arias-liménez JL, Llàcer-lborra P, et al. Heart failure with mid-range ejection fraction in patients admitted to internal medicine departments: Findings from the RICA Registry. Int J Cardiol. 2018 Mar;255:124-8.

27. Lund LH, Claggett B, Liu J, Lam CS, Jhund PS, Rosano GM, et al. Heart failure with mid-range ejection fraction in CHARM: characteristics, outcomes and effect of candesartan across the entire ejection fraction spectrum. Eur J Heart Fail. 2018;20(8):1230-9.

28. Johansson I, Dahlström U, Edner M, Näsman P, Rydén L, Norhammar A. Type 2 diabetes and heart failure: Characteristics and prognosis in preserved, mid-range and reduced ventricular function. Diab Vasc Dis Res. 2018;15(6):494-503.

29. Moliner P, Lupón J, Barallat J, de Antonio M, Domingo M, Núñez J, et al Bio-profiling and bio-prognostication of chronic heart failure with mid-range ejection fraction. Int J Cardiol. 2018 Apr;257:188-92.

30. Rastogi A, Novak E, Platts AE, Mann DL. Epidemiology, pathophysiology and clinical outcomes for heart failure patients with a mid-range ejection fraction. Eur J Heart Fail. 2017;19(12):1597-605.

31. Chioncel O, Lainscak M, Seferovic PM, Anker SD, Crespo-Leiro MG, Harjola $\mathrm{VP}$, et al. Epidemiology and one-year outcomes in patients with chronic heart failure and preserved, mid-range and reduced ejection fraction: an analysis of the ESC Heart Failure Long-Term Registry. Eur J Heart Fail. 2017;19(12):1574-85.

32. Altaie $\mathrm{S}$, Khalife W. The prognosis of mid-range ejection fraction heart failure: a systematic review and meta-analysis. ESC Heart Fail. 2018;5(6):1008-16

33. Solomon SD, Claggett B, Lewis EF, Desai A, Anand I, Sweitzer NK, et al. Influence of ejection fraction on outcomes and efficacy of spironolactone in patients with heart failure with preserved ejection fraction. Eur Heart 2016;37(5):455-62.

34. Xin Y-G, Chen X, Zhao Y-N, Hu J, Sun Y, Hu WY. Outcomes of spironolactone treatment in patients in Northeast China suffering from heart failure with mid-range ejection fraction. Curr Med Res Opin. 2019;35(4):561-8.

35. Hernandez AF, Hammill BG, O'Connor CM, Schulman KA, Curtis LH, Fonarow GC. Clinical effectiveness of beta-blockers in heart failure: findings from the OPTIMIZE-HF (Organized Program to Initiate Lifesaving Treatment in Hospitalized Patients with Heart Failure) Registry. J Am Coll Cardiol 2009;53(2):184-92.

36. Konstam MA. Seeking therapeutic precision in heart failure: is ejection fraction really the way? Deconstructing the CHARM of heart failure with mid-range ejection fraction. Eur J Heart Fail. 2018;20(8):1240-2.

37. Cleland JG, Bunting KV, Flather MD, Altman DG, Holmes J, Coats AJ, et al. Beta-blockers for heart failure with reduced, mid-range, and preserved ejection fraction: an individual patient-level analysis of double-blind randomized trials. Eur Heart J. 2018;39(1):26-35.

38. Gwag HB, Lee GY, Choi JO, Lee HY, Kim JJ, Hwang KK, et al. Fate of Acute Heart Failure Patients With Mid-Range Ejection Fraction. Circ J. 2018;82(8):2071-8.

39. Abdul-Rahim AH, Shen L, Rush CJ, Jhund PS, Lees KR, McMurray JJ, et al. Effect of digoxin in patients with heart failure and mid-range (borderline) left ventricular ejection fraction. Eur J Heart Fail. 2018;20(7):1139-45.

40. Dunlay SM, Roger VL, Weston SA, Jiang R, Redfield MM. Longitudinal changes in ejection fraction in heart failure patients with preserved and reduced ejection fraction. Circ Heart Fail. 2012;5(6):720-6.

41. Ather S, Chan W, Bozkurt B, Aguilar D, Ramasubbu K, Zachariah AA, et al. Impact of noncardiac comorbidities on morbidity and mortality in a predominantly male population with heart failure and preserved versus reduced ejection fraction. J Am Coll Cardiol. 2012;59(11):998-1005.

42. Zinman B, Wanner C, Lachin JM, Fitchett D, Bluhmki E, Hantel S, et al. Empagliflozin, Cardiovascular Outcomes, and Mortality in Type 2 Diabetes. N Engl J Med. 201;373(22):2117-28. 